\title{
Respiratory Microbiota and Lower Respiratory Tract Disease
}

Introduction: The respiratory airways harbor a complex succession of ecological niches with distinct but related bacterial communities. Particular challenges of respiratory microbiome research have led to limited scientific output compared to other human microbiomes.

Areas covered: In this review, we summarize the current state of knowledge of the bacterial respiratory microbiome, with a particular focus on associations between the respiratory microbiome and lower respiratory tract conditions.

Expert commentary: There is growing evidence that the respiratory microbiome is associated with lower respiratory infectious diseases and related conditions. Most respiratory microbiome reports are metataxonomic cross-sectional or case-control studies with relatively small sample sizes. Large, prospective projects with metatranscriptomics or metabolomics approach are needed to unravel the effect of the respiratory microbiome on health-related conditions. Moreover, standardization in sampling, library preparation, sequencing techniques and data analysis should be encouraged. 


\section{Introduction}

In the past decade, we have witnessed an exponential increase in human microbiome related research, from around 300 publications in 2006 to almost 8000 in 2016 . The development of novel culture-independent techniques of microbial identification, and decreasing costs of sequencing platforms have enabled research in this exciting field, producing relevant findings that have changed and broaden our perspective on hostmicrobe and microbe-microbe interactions. Important paradigm shifts have occurred during this process, including the recognition of the diversity and abundance of the microbial community in healthy individuals, the discovery of viable communities in body sites previously considered sterile, and more importantly, the establishment of associations between human microbiome and health.

Most of the research conducted so far has focused on the gut microbiota, the body site containing the richest and most complex bacterial communities. In 2008, the National Institutes of Health and the European Commission launched two multicentric mega projects: the Human Microbiome Project, where samples from up to 18 body sites of healthy individuals were collected, and MetaHIT, focused on the metagenomics of the intestinal tract. ${ }^{1,2}$ The respiratory tract was left apart, partly because of problems in collecting lower respiratory tract samples, but also because lungs have traditionally been considered sterile. Recent studies have shown that the lower respiratory tract harbours a diverse bacterial community both in healthy and diseased individuals, which has boosted respiratory microbiota research that is currently growing rapidly. ${ }^{3}$

Acute lower respiratory infections, such as pneumonia and bronchiolitis are a major public health concern globally, accounting for a large burden of mortality and hospitalization in children. ${ }^{4-6}$ Chronic conditions, such as asthma and chronic obstructive pulmonary disease (COPD), show a high and growing prevalence globally, with respiratory infections being involved in pathogenesis and course of the disease. ${ }^{7-}$

10 Respiratory microbiota research may reveal new perspectives regarding hostmicrobe interactions leading to a particular local environment contributing to the pathogenesis of lung and airways conditions. These deeper insights could later be the basis for novel preventive and therapeutic measures to complement or adapt the currently available arsenal against respiratory infectious diseases.

Besides an initial overview of respiratory microbiota composition and dynamics, this review aims to summarize published evidence of associations between respiratory microbiota and different respiratory conditions.

\section{Terminology and methods}

Most microbiota studies rely on sequencing variable regions of the highly conserved gene encoding the 16 small subunit of ribosomal RNA (16SrRNA). This gene is universally present in bacteria and absent in mammals, and contains 9 hypervariable regions (V1 to V9) allowing the identification of different bacteria through taxonomic assignment of the obtained sequences using reference genomes. ${ }^{11}$ Depending on the sequences and the available databases, identification can be achieved to the species level but usually results in a mix of species, genera, phylum, and taxa levels. To 
describe the output of microbial identification, the generic term organizational taxonomic unit (OTU) is usually adopted. This process is defined as metataxonomics as it describes the taxonomic composition of the entire bacterial community. This has two important implications: first, viruses and fungus (which lack the 16S rRNA gene although similar profiling methods based on the 18S rRNA gene are available for fungus) are not identified albeit being an integral part of the microbiota, and second, this process does not provide information about the function of the microbiota, which can only be gained through metagenomics or other omics platforms, such as metatranscriptomics and metabolomics. Key terms used in microbiota research are defined in Table 1.

\section{Ecological niches and dynamics of the respiratory microbiota}

\subsection{Microbiota acquisition of the upper respiratory tract}

From a bacterial ecological perspective, the human respiratory tract is a sequence of niches from the anterior nares and mouth to the lungs harbouring distinct but related bacterial communities. There is recent evidence of foetal microbial colonization starting in utero, ${ }^{12,13}$ with intensification of the process immediately after birth. Maternal bacteria rapidly colonize cutaneous and mucosal surfaces of the newborn, with predominance of vaginal and intestinal maternal microbiota in case of vaginal delivery, and of skin microbiota in births resulting from caesarean sections. ${ }^{14}$ The anterior nares display a relatively simple microbiota with few differences between children and adults. Corynebacterium, Propionibacterium and Staphylococcus are the initial colonizers of the anterior nares and maintain their predominance until adult life where Moraxella has also been described together with the three genera above. ${ }^{15}$ In contrast, nasopharyngeal microbiota is a more complex and dynamic ecosystem with important changes over time. Differentiation into site-specific microbiota is a multifactorial process that starts after initial colonization and can last to up to two years of age depending on body sites, with the upper respiratory tract microbiota of children 18 months of age still showing distinct characteristics compared to that of adults. ${ }^{16,17}$ Initially, Corynebacterium and Staphylococcus dispersing from the anterior nares colonize the nasopharynx that subsequently gains Dolosigranulum (particularly in breastfed infants), Alloiococcus and Moraxella. ${ }^{18-20}$ In adults, the inter-individual diversity in nasopharyngeal microbiota is reduced, Corynebacterium is usually the dominant genus, and Moraxella and Dolosigranulum are much less frequently represented. ${ }^{21-23}$ The oral cavity shows the same initial colonization strongly influenced by delivery mode as the other body sites, but rapidly gains a higher richness due to a high exposure to the external environment, the presence of saliva characterized by abundance of Prevotella, Neisseria, and Haemophilus, and later on, by the eruption of teeth entailing an increase in Bacteroidetes, particularly Veillonella and Prevotella. ${ }^{24}$ Despite being highly exposed to foodborne and environmental bacteria, the oral microbiota is remarkably stable over time. ${ }^{25}$ The oropharynx is an anatomical crossroad between the upper respiratory structures, and the lower airways and gastrointestinal tract resulting in a rich and highly variable bacterial ecosystem. Common genera described in healthy adults include Streptococcus (both commensal and potentially pathogenic such as $S$. pneumoniae and S. pyogenes), Haemophilus, Neisseria, Prevotella, Veillonella and Leptotrichia. ${ }^{26,27}$ Similar to what happens in the 
gut mucosa and skin surface, the early colonization of upper airways plays an important role in immune maturation and immune response modulation of the host, as suggested in studies where germ-free mice showed exaggerated airway eosinophilia and accumulation of invariant natural killer T-cells that did not occur in colonized mice. ${ }^{28-31}$ Initially, the neonate immune system is skewed towards a regulatory response allowing microbiota colonization without inflammatory response. The risk of having an immature immune system at birth is rapidly compensated by the beneficial effect of having a stable commensal microbiota conferring resistance to colonization by pathogenic bacteria. The contact between resident microbiota and the infant immune system is limited by physical barriers such as mucus layers, and modulated by metabolites, microbes, and IgA contained in maternal milk, leading to immunogenic tolerance and the establishment of a stable host-microbiota relationship. ${ }^{32}$ Despite the virtually absent inflammatory response against stable microbiota, the constant exposure to limited amounts of bacterial antigens contributes to the development of the immune system.

\subsection{The lower respiratory tract microbiota}

The healthy lung sterility paradigm has repeatedly been challenged since the introduction of culture-independent techniques. However, this does not imply the existence of stable colonization of the lungs and it is commonly accepted that bacterial detection in lower airways samples is likely due to equilibrium between constant inoculation of upper respiratory microbiota by micro-aspiration, and clearance by respiratory endothelium cilia and cough. ${ }^{33-35}$ Venkataraman et al. showed microbial composition of the healthy lung in adults was consistent with the neutral community model, i.e., bacterial dispersal from upper respiratory niches, particularly the oral cavity, explained lung microbial communities. ${ }^{36}$ Interestingly, viable bacteria account for $61 \%$ of the recovered OTUs. In diseased lungs, however, selection seemed to shape bacterial communities, suggesting stable colonization. In healthy lungs, bronchioles and alveoli are covered with surfactant and its low content in nutrients impair bacterial growth. ${ }^{37}$ In case of infection, the high protein content exudate resulting from inflammatory response serves as substrate for bacterial overgrowth. Studies have shown the composition of the microbiome of the lower airways in healthy adults to correlate with that of the upper airways, particularly the oropharynx, but to be much lower in abundance (from $10^{5}$ bacteria per milliliter of nasopharyngeal fluid to $10^{1}$ $10^{2} / \mathrm{mL}$ of BAL fluid). ${ }^{38-41}$ Similar results have been obtained in children, except for a greater contribution of nasopharyngeal microbiota in lower airways' microbiota composition. ${ }^{42}$ Segal et al. partially challenge these findings. In two cross-sectional studies with healthy adults, they determined two distinct pneumotypes based on bronchoalveolar lavage (BAL) samples, one enriched with bacteria from the upper respiratory tract and present in around $45 \%$ of participants, and the other enriched with background bacteria. ${ }^{43,44}$ The first pneumotype was associated with increased pulmonary inflammation measured by exhaled nitric oxide and BAL cells count. This subclinical inflammation characterized by increased Th17 response might be beneficial for the host, as suggested by animal models of Staphylococcus aureus pneumonia. ${ }^{45}$ 


\section{Respiratory microbiota and lower respiratory conditions}

We systematically reviewed the literature to retrieve original papers describing associations between respiratory microbiota and lower respiratory tract infections (Box 1). We excluded HIV infected patients, as it was object of a recently published review. ${ }^{3}$ A total of 25 articles were retained for this section, 13 in adults, ${ }^{46-58}$ and 11 in children. ${ }^{19,59-69}$ An overview of the studies characteristics is summarized in tables 2 (adults) and 3 (children).

\subsection{Pneumonia and ventilator-associated pneumonia}

Despite differences in study populations and samples, similar patterns have been observed in patients with pneumonia. In general, such patients display a more abundant microbiota with less diversity, richness and evenness, and these disturbances can be observed in upper and lower respiratory tract microbiota, particularly in adults. The microbiota of patients with pneumonia seems to be dominated by one taxon, showing a higher relative abundance of Streptococcus, Haemophilus or Moraxella. Dominance by Streptococcus pneumoniae, Burkholderia, Bacillales, and to a lesser extent, Pseudomonadales was particularly correlated with decreased diversity. ${ }^{58,68}$ In studies with repeated sampling, the dominance of one taxon was maintained over time, and its relative abundance increased throughout the pneumonia episode, particularly if the patient was intubated. ${ }^{51}$ Intubation was consistently a risk factor for bacterial disturbance in the lower respiratory tract (low diversity and evenness) supporting the hypothesis of a lung microbiota resulting from the equilibrium between microaspirations from the upper respiratory tract and clearance mechanisms. ${ }^{34,51,58,63}$ Antibiotic administration seemed to have less effect on bacterial diversity than intubation, ${ }^{58}$ and this effect was transitory, with recovery of a similar oropharyngeal microbiota a few days after antibiotic treatment compared to the microbiota before antibiotic administration. ${ }^{52}$ However, most of the studies did not assess this effect, probably due to difficulties in assuming bacterial viability from DNA based measures. ${ }^{70,71}$ In an early study assessing the effect of antibiotics in seven intubated adults colonized by Pseudomonas aeruginosa, Shannon's diversity index significantly fell from 1.48 to 0.59 after a few days of antibiotic treatment, with $P$. aeruginosa coming to dominate the microbiome in most patients despite susceptibility in vitro to the administered antibiotics. ${ }^{72}$ No taxon was consistently associated with health, although some associations were found. Prevotella dominant oropharyngeal microbiota was more frequent in healthy adults than in adults with pneumonia, but more frequent in older children with severe pneumonia than in non-severe pneumonia cases. $^{46,66}$ Veillonella and Dolosigranulum were frequently sequenced in healthy individuals or patients with less severe pneumonia in upper respiratory tract samples, the latter particularly in young children. ${ }^{46,66,67,73}$ Interestingly, despite Moraxella being associated with pneumonia, Moraxella lacunata was significantly more frequent in pediatric viral vs. non-viral pneumonia, and more frequent in controls than in all-cause pneumonia, highlighting the necessary caution in establishing associations based on phylum or genera-level taxonomics. ${ }^{67}$

Interesting inconsistencies merit further examination. In a study in adults with community-acquired pneumonia and healthy controls, oropharyngeal microbiota dominated by Lactobacillales (predominantly Streptococcus spp.) was associated with 
pneumonia. ${ }^{46}$ This result was partly consistent with a pediatric study assessing the association between upper and lower respiratory tract microbiota and pneumonia severity. ${ }^{66}$ Presence of Lactobacillales was associated with longer hospital stay in children older than five years of age, but with a less severe pneumonia in under-five children underscoring the importance of age-related host-microbe interactions and immune system dynamics. Two studies did not support the association between low microbiota diversity and disease. Toma et al. compared the microbiota of endotracheal aspirates from intubated adults with possible ventilator-associated pneumonia and found no differences in diversity when comparing low and high-risk groups as per the Clinical Pneumonia Infection Score (CPIS) ${ }^{56}$ However, there were no significant differences between groups in most of the items included in the CPIS, and the authors did not report clinical outcomes at the end of the admission, raising concerns of misclassification. Wang et al. compared the microbiota of bronchoalveolar lavage fluid in children with pneumococcal and Mycoplasma pneumonia with controls, and reported that controls had the lowest microbial diversity. ${ }^{68}$ This could be evidence of age-dependent differences in health and disease, although caution is warranted at this stage of microbiome research. Noteworthy, controls $(n=11)$ were younger than cases and had tracheomalacia, which may play a role in impaired clearance of the lower respiratory tract in individuals from an age group with a respiratory microbiota yet to be fully developed.

\subsection{Bronchiolitis and asthma}

Recurrent wheezing and asthma in childhood are strongly associated with having suffered a bronchiolitis episode during the first year of life, particularly when caused by respiratory syncytial virus (RSV) or human rhinovirus (HRV). We retrieved six original papers assessing the respiratory microbiota in infants with bronchiolitis, and one in children and adolescents with asthma, with all of them using upper airways samples. ${ }^{19,59-62,64,69}$ The article on asthma was included because it focused on the infectious component of recurrent wheezing pathophysiology. Despite a smaller variability in study population and in sampling sites than in the aforementioned pneumonia studies, findings in this condition are discordant. While there is some degree of consensus in describing a decreased evenness in both asthma and bronchiolitis, discrepancies arise on which phylum or genus dominates the microbiota. Most studies associate Proteobacteria (phylum) and Moraxella or Haemophilus (genera) dominant taxa in upper respiratory microbiota with asthma, bronchiolitis or severity of bronchiolitis. ${ }^{19,59,62,69}$ On the other hand, in a case-control study with infants with bronchiolitis and healthy controls, Hasegawa et al. described four distinct profiles of upper respiratory microbiota, each of them dominated by a taxon, with infants with the Moraxella dominant profile having the lowest risk of having bronchiolitis, and the Staphylococcus dominant profile presenting an odds ratio of 5 for bronchiolitis. ${ }^{60}$ Interestingly, Dolosigranulum dominant profile also presented a very low risk of bronchiolitis, consistent with the findings we described above in the section dedicated to pneumonia. In another large case-series published by the same author assessing the association of microbiota and bronchiolitis severity, Moraxella dominant profiles presented again the lowest risk of a negative outcome (defined as admission to the intensive care unit). ${ }^{61}$ In this series, the profile with highest risk of negative outcome was the Haemophilus dominant one, consistent with most of the studies. Seasonal or geographical effects do not explain these discrepancies as most of the studies were 
conducted in the US in winter and merit further investigation. Four studies reported data on viral etiology and suggested virus-bacteria interactions in the pathogenesis of bronchiolitis and future recurrent wheezing, particularly between Proteobacteria and RSV. ${ }^{19,62,69}$ The chronology of the interaction, i.e., whether a viral infection alters the epithelium environment allowing for a greater bacterial adherence or a colonizing bacteria predisposes to viral infection, is still unclear, although there is some evidence supporting the former at least in case of human rhinovirus infection (hRV). In a prospective study with weekly nasal samples collected from children aged four to twelve years old, detection of hRV increased the risk of concurrent or subsequent detection of pathogenic bacteria (either $S$. pneumoniae, $H$. influenzae or $M$. catharralis), whereas the opposite was not observed. ${ }^{74}$ In a randomized controlled trial in infants with RSV-bronchiolitis admitted to hospital, Zhou et al. assessed the effect of a 14-days course of azithromycin (AZM) on future recurrent wheezing, reporting a $50 \%$ reduction over the following 12 months. ${ }^{69}$ Nasal lavages samples were taken before and after the treatment showing a significantly higher reduction in Moraxella relative abundance in the treatment group. Cautiously, the authors recognized difficulties unfolding the direct anti-inflammatory effect of AZM on respiratory airways and the indirect effect through Moraxella depletion on the recurrent wheezing reduction. On the other hand, Mansbach et al. found that in infants hospitalized with severe bronchiolitis, RSV mono-infection was associated with high relative abundance of Firmicutes (Streptococcus) and low abundance of Proteobacteria (Moraxella and Haemophilus), with an inverse association in HRV mono-infection. ${ }^{64}$ To note, this caseseries is the same as the one described by Hasegawa et al.

\subsection{Bronchiolitis obliterans syndrome (BOS)}

Lung transplant recipients face a higher risk of acute and chronic rejection of the allograft than other solid organ recipients, with BOS being the leading cause of death after the first year. ${ }^{75}$ Although epidemiologically less relevant than pneumonia and asthma, the dramatic outcome of BOS and the host-microbe interactions involved merit a closer attention. Traditionally, proper immunosuppression and aggressive antibiotic treatment, particularly against Pseudomonas aeruginosa have been the main pillars in BOS prevention and management. ${ }^{76}$ Case-control studies using cultureindependent techniques are providing notable insight that may change our perception of BOS pathogenesis. ${ }^{47,57}$ Lung transplant patients harbor a more abundant and less diverse lower airways microbiota than non-transplant patients. Two main pneumotypes have been described according to genera dominance, one dominated by Pseudomonas and the other dominated by Veillonella, Prevotella or Streptococcus. These pneumotypes seem to be mutually exclusive, and although Pseudomonas abundance is associated with low diversity, which is in turn associated with higher BAL neutrophilia and bacterial DNA burden, assigning a healthy or unhealthy label to these pneumotypes is not straightforward. Dickson et al. assessed the association between BAL microbiota and BOS in 33 adults with lung transplant, and identified an uncommon Pseudomonas species, Pseudomonas fluorescens. ${ }^{47}$ Almost all the patients with $P$. aeruginosa had symptoms associated with BOS, whereas all the patients with $P$. fluorescens were symptom-free. A case-control study with 57 lung transplant recipients and 8 controls conducted by Willner et al. yielded results requiring a close attention. ${ }^{57}$ Half of the lung transplant recipients had cystic fibrosis (CF), with all of them being colonized by $P$. aeruginosa prior to transplantation. In this group, 
recolonization by $P$. aeruginosa, usually from upper airways reservoirs, was negatively associated with BOS (OR 0.25; 95\% confidence interval 0.09-0.65), whereas presence of newly acquired $P$. aeruginosa was associated with developing BOS in the non-CF subgroup. These results might partly be explained by the phenotypic changes suffered by $P$. aeruginosa, transitioning from an aggressive form with high expression of virulence factors when acquired de novo to a more passive phenotype after long-term colonization showing less invasiveness and thus eliciting immunogenic response. ${ }^{77}$ The authors conclude the reestablishment of pre-transplant populations in the allograft appear to protect against BOS, which supports the hypothesis of immunogenic tolerance in airways inflammation.

\subsection{Others}

This section includes a miscellanea of lower respiratory tract conditions in adults, from non-transplanted cystic fibrosis and non-CF bronchiectasis to interstitial lung disease and idiopathic pulmonary fibrosis, all of them directly or indirectly associated with infection. ${ }^{48,49,53,54}$ Three studies assessed the association between respiratory microbiota and lung function, measured by spirometry, and found a correlation between microbiota diversity and forced expiratory volume in 1 second (FEV1). ${ }^{48,53,54}$ In one of these studies, however, the lung function deterioration seemed to be driven more by bacterial abundance than microbiota diversity or composition. ${ }^{53}$ Patients with idiopathic pulmonary fibrosis had slightly lower diversity than controls (Shannon index 3.8 vs. 4.1) and similar microbiota composition with Streptococcus, Prevotella and Veillonella accounting for $50 \%$ of reads in both groups, but significantly higher abundance than controls. These findings were also reported in a sub-group analysis comparing progressors and non-progressors among cases. To note, controls included patients with moderate COPD, which is associated with lower microbiota diversity. ${ }^{78}$ On the other hand, Garzoni et al. compared the microbiota composition and diversity in upper and lower respiratory tract samples from 24 patients with interstitial lung disease or Pneumocystis jirovecii pneumonia and 9 healthy controls, and found no differences in richness, diversity and presence of commensals such as Prevotella or Acidaminococcaceae suggesting no effect of respiratory microbiota in the disease process. ${ }^{49}$ The small sample size and the variety of diagnoses in the disease group could arise concerns about the internal validity of this study, but if confirmed, these negative results could help researchers in narrowing the pathophysiology of hostmicrobe interactions in lung disease.

\section{Conclusion}

Despite the variability of study populations, samples and methods used in the original papers included in this review, patterns in the effect of respiratory microbiota on lower respiratory tract infections and infection-related conditions start to arise, although a clear healthy respiratory microbiota is still to be defined. Including metagenomics and metabolomics data to metataxonomics evidence accumulated to date could shed some light to the field, although hypothesis-driven research should be strongly encouraged to minimize the risk of getting "lost in transcription". 


\section{Expert commentary}

Interpreting and comparing respiratory microbiota data across studies is a difficult task. The main issue when studying the respiratory microbiota compared to the gut microbiota is the invasiveness of lung sampling. Some attempts to overcome this have been suggested, such as combining nasopharyngeal and oropharyngeal samples based on the relationship between these sites and the lower respiratory tract microbiota, and an interesting assessment of the lung microbiota through exhaled breath condensate fluid that showed a high correlation with bronchoalveolar lavage fluid in intubated patients ${ }^{79}$, although this technique does not seem to be easily applicable to non-intubated patients. Besides the difference in samples, variability in sequencing techniques, sequenced regions, library preparation, bioinformatics tools and reference databases among others could yield different results. Kumar et al., for example, reported differences in taxonomic results when sequencing different variable regions of the 16SrRNA gene and suggested that V1 to V3, and V7 to V9 showed the greatest utility in classification. ${ }^{80}$ On the other hand, Kelly et al. reported similar results when comparing the performance of two sequencing platforms (Illumina MiSeq and Roche/454) on the same samples. ${ }^{51}$ To avert these issues, it is necessary to standardize existing techniques, to keep establishing associations between the microbiota from different sampled respiratory sites, and ideally, to create a unique genome reference database. In addition, molecular biology tools to assess viability of bacteria identified by sequencing methods should be applied when possible. This would help reduce the intricacy of microbiota projects' results and establishing patterns, a key step to move from the observational nature of the microbiota studies to date to hypothesis driven and interventional projects.

Most of the studies assessing associations between the respiratory microbiota and disease are metataxonomic studies (i.e., based on 16SrRNA sequencing) with relatively small sample sizes. Even with statistical corrective measures to account for multiple comparisons, spurious associations cannot completely be ruled out. Multiplicity of comparisons is an intrinsic issue in microbiota research because of the myriad of bacterial species co-existing in the human body, and the multiple characteristics we use to describe bacterial communities, i.e., evenness/dominance, richness, abundance and presence of specific taxa. However, there appears to be a certain consistency in microbiota studies where diversity is usually associated with health, and dominance of a single species usually associated with disease. Importantly, these could just be proxies of respectively presence and absence of a particular bacterial consortium creating a specific environment with protective or beneficial effect. This hypothesis is supported by some studies with animal models showing that resistance against Clostridium difficile colonization was not conferred by any single bacterial population, but rather by combinations of different commensals. ${ }^{81}$

Moreover, the dynamics and the effect over time of the respiratory microbiota have scarcely been studied raising reasonable doubts about the causal relationship between findings in microbiota composition and disease. Besides the cohort study conducted by Teo et al. and described in the "Asthma" section, another cohort study led by Santee et al. showed that dominance of either Moraxella, Streptococcus or Haemophilus in the nasopharynx of children aged 4 to 7 years was associated with more frequent 
upper respiratory tract infections including sinusitis over a 1-year follow-up. ${ }^{82}$ These findings should encourage researchers to conduct cohort studies in humans and interventional studies in animal models to validate the results from cross-sectional studies as a necessary step towards designing preventive measures against respiratory conditions based on the respiratory microbiota. Ideally, these potential novel measures should be applicable in developing countries, the world's region that suffers the highest burden of pediatric respiratory infections, and that is undergoing an epidemiological transition towards a double burden of disease with an important role of chronic respiratory conditions.

\section{Five-years view}

Currently, the dominance of host transcripts and metabolites in respiratory samples obscures the interpretation of costly transcriptome and metabolome studies. With decreasing costs of culture-independent techniques, particularly whole genome sequencing, innovative solutions to overcome interpretation issues of results from omic platforms, and full integration with other disciplines (e.g., immunology, bioinformatics, microbiology, epidemiology), the bacterial microbiota research is likely to become a true pan-microbiome field with a comprehensive understanding of bacterial, viral, fungal and host interactions. The development of biobanks are a good ally for this endeavor, insofar it will enable analyzing large numbers of stored samples once the costs of omic platforms decrease exponentially. This will constitute the basics towards interventional research where probiotics, prebiotics and nutrition will probably be joined by phages and extremely narrow spectrum antibiotics as key interventions to build a healthy respiratory microbiota to be used as a preventive and therapeutic measure. Taking into account environmental effects, population and individual diversity, cross-talk between microbial communities from different body sites, and the effects of the microbiota on different diseases and conditions, the microbiome research could become one of the pillars of personalized medicine.

\section{Key issues}

- The respiratory tract is a complex ecosystem with distinct bacterial communities present in every niche, including the lungs.

- The respiratory microbiota seems to have an effect on lower respiratory infections and infectious-related conditions, where host-microbe and microbemicrobe interactions are not yet completely understood.

- Although microbiota diversity is usually associated with health, it is unclear whether diversity may mask the presence of protective bacterial consortia. Moreover, differences in children and adults might be evidence of important interactions associated with immune system development.

- Prospective studies with serial samples to describe the respiratory microbiome acquisition and dynamics, and to determine long-term effects on respiratory conditions are needed to exploit the translational potential of the respiratory microbiome. 


\section{Funding}

This work was supported by Fundação para a Ciência e a Tecnologia (FCT, Portugal) grant to GHTM - UID/Multi/04413/2013, and by "Plan Nacional de I+D+l" and "Instituto de Salud Carlos III- Subdirección General de Evaluación y Fomento de la Investigación Sanitaria", project PI16/00174, and the European Regional Development Fund (FEDER). ISGlobal is a member of the CERCA Programme, Generalitat de Catalunya.

\section{Declaration of interest}

The authors have no relevant affiliations or financial involvement with any organization or entity with a financial interest in or financial conflict with the subject matter or materials discussed in the manuscript.

\section{References}

1. Blanco-Miguez A, Gutierrez-Jacome A, Fdez-Riverola F, Lourenco A, Sanchez B. MAHMI database: a comprehensive MetaHit-based resource for the study of the mechanism of action of the human microbiota. Database (Oxford) 2017; 2017.

2. Turnbaugh PJ, Ley RE, Hamady M, Fraser-Liggett CM, Knight R, Gordon JI. The human microbiome project. Nature 2007; 449(7164): 804-10.

3. Lawani MB, Morris A. The respiratory microbiome of HIV-infected individuals. Expert Rev Anti Infect Ther 2016; 14(8): 719-29.

4. Bont L, Checchia PA, Fauroux B, et al. Defining the Epidemiology and Burden of Severe Respiratory Syncytial Virus Infection Among Infants and Children in Western Countries. Infect Dis Ther 2016; 5(3): 271-98.

5. Nair H, Nokes DJ, Gessner BD, et al. Global burden of acute lower respiratory infections due to respiratory syncytial virus in young children: a systematic review and meta-analysis. Lancet 2010; 375(9725): 1545-55.

6. Walker CL, Rudan I, Liu L, et al. Global burden of childhood pneumonia and diarrhoea. Lancet 2013; 381(9875): 1405-16.

7. Adeloye $D$, Chua $S$, Lee $C$, et al. Global and regional estimates of COPD prevalence: Systematic review and meta-analysis. J Glob Health 2015; 5(2): 020415.

8. Asher I, Pearce N. Global burden of asthma among children. Int J Tuberc Lung Dis 2014; 18(11): 1269-78.

9. King PT, MacDonald M, Bardin PG. Bacteria in COPD; their potential role and treatment. Transl Respir Med 2013; 1(1): 13.

10. Bisgaard $\mathrm{H}$, Hermansen $\mathrm{MN}$, Buchvald $\mathrm{F}$, et al. Childhood asthma after bacterial colonization of the airway in neonates. N Engl J Med 2007; 357(15): 1487-95.

11. Beck JM. ABCs of the lung microbiome. Ann Am Thorac Soc 2014; 11 Suppl 1: S3-6. 
12. Romano-Keeler J, Weitkamp JH. Maternal influences on fetal microbial colonization and immune development. Pediatr Res 2015; 77(1-2): 189-95.

13. Stinson LF, Payne MS, Keelan JA. Planting the seed: Origins, composition, and postnatal health significance of the fetal gastrointestinal microbiota. Crit Rev Microbiol 2017; 43(3): 352-69.

14. Dominguez-Bello MG, Costello EK, Contreras M, et al. Delivery mode shapes the acquisition and structure of the initial microbiota across multiple body habitats in newborns. Proc Natl Acad Sci U S A 2010; 107(26): 11971-5.

15. Zhou Y, Mihindukulasuriya KA, Gao H, et al. Exploration of bacterial community classes in major human habitats. Genome Biol 2014; 15(5): R66.

16. Perez-Losada M, Alamri L, Crandall KA, Freishtat RJ. Nasopharyngeal Microbiome Diversity Changes over Time in Children with Asthma. PLoS One 2017; 12(1): e0170543.

17. Bogaert D, Keijser B, Huse $S$, et al. Variability and diversity of nasopharyngeal microbiota in children: a metagenomic analysis. PLoS One 2011; 6(2): e17035.

18. de Steenhuijsen Piters WA, Sanders EA, Bogaert D. The role of the local microbial ecosystem in respiratory health and disease. Philos Trans $R$ Soc Lond B Biol Sci 2015; 370(1675).(pii): 20140294. doi: 10.1098/rstb.2014.0294.

19. Teo SM, Mok D, Pham K, et al. The infant nasopharyngeal microbiome impacts severity of lower respiratory infection and risk of asthma development. Cell Host Microbe 2015; 17(5): 704-15. doi: 10.1016/j.chom.2015.03.008. Epub Apr 9.

** This is a cohort study with a 5 -year follow-up to assess the association between early-life nasopharyngeal microbiome and risk of developing asthma in childhood. 20. Biesbroek $G$, Bosch AA, Wang $X$, et al. The impact of breastfeeding on nasopharyngeal microbial communities in infants. Am J Respir Crit Care Med 2014; 190(3): 298-308.

21. Hilty $\mathrm{M}$, Burke $\mathrm{C}$, Pedro $\mathrm{H}$, et al. Disordered microbial communities in asthmatic airways. PLoS One 2010; 5(1): e8578.

22. Stearns JC, Davidson CJ, McKeon S, et al. Culture and molecular-based profiles show shifts in bacterial communities of the upper respiratory tract that occur with age. ISME J 2015; 9(5): 1268.

23. Allen EK, Koeppel AF, Hendley JO, Turner SD, Winther B, Sale MM.

Characterization of the nasopharyngeal microbiota in health and during rhinovirus challenge. Microbiome 2014; 2: 22.

24. Crielaard W, Zaura E, Schuller AA, Huse SM, Montijn RC, Keijser BJ. Exploring the oral microbiota of children at various developmental stages of their dentition in the relation to their oral health. BMC Med Genomics 2011; 4: 22.

25. Zaura E, Nicu EA, Krom BP, Keijser BJ. Acquiring and maintaining a normal oral microbiome: current perspective. Front Cell Infect Microbiol 2014; 4: 85.

26. Gong $\mathrm{H}$, Shi $\mathrm{Y}$, Zhou $X$, et al. Microbiota in the Throat and Risk Factors for Laryngeal Carcinoma. Appl Environ Microbiol 2014; 80(23): 7356-63.

27. Segata N, Haake SK, Mannon P, et al. Composition of the adult digestive tract bacterial microbiome based on seven mouth surfaces, tonsils, throat and stool samples. Genome Biol 2012; 13(6): R42.

28. Gollwitzer ES, Saglani S, Trompette A, et al. Lung microbiota promotes tolerance to allergens in neonates via PD-L1. Nat Med 2014; 20(6): 642-7. 
29. Olszak T, An D, Zeissig S, et al. Microbial exposure during early life has persistent effects on natural killer T cell function. Science 2012; 336(6080): 489-93.

30. Belkaid $\mathrm{Y}, \mathrm{H}$ and TW. Role of the microbiota in immunity and inflammation. Cell 2014; 157(1): 121-41.

31. Man WH, de Steenhuijsen Piters WA, Bogaert D. The microbiota of the respiratory tract: gatekeeper to respiratory health. Nat Rev Microbiol 2017.

32. Marcobal A, Sonnenburg JL. Human milk oligosaccharide consumption by intestinal microbiota. Clin Microbiol Infect 2012; 18 Suppl 4: 12-5.

33. Dickson RP, Erb-Downward JR, Huffnagle GB. The role of the bacterial microbiome in lung disease. Expert Rev Respir Med 2013; 7(3): 245-57.

34. Dickson RP, Erb-Downward JR, Huffnagle GB. Towards an ecology of the lung: new conceptual models of pulmonary microbiology and pneumonia pathogenesis.

Lancet Respir Med 2014; 2(3): 238-46. doi: 10.1016/S2213-600(14)70028-1.

* This article proposes new conceptual models constituting interesting frameworks to design respiratory microbiota projects and interpret future findings.

35. Taylor SL, Wesselingh S, Rogers GB. Host-microbiome interactions in acute and chronic respiratory infections. Cell Microbiol 2016; 18(5): 652-62. doi:

10.1111/cmi.12589.

36. Venkataraman A, Bassis CM, Beck JM, et al. Application of a neutral community model to assess structuring of the human lung microbiome. MBio 2015; 6(1).

* This article applies different ecological models to respiratory samples in order to explain the acquisition and dynamics of the lung microbiota.

37. Fahy JV, Dickey BF. Airway mucus function and dysfunction. N Engl J Med 2010; 363(23): 2233-47.

38. Bassis CM, Erb-Downward JR, Dickson RP, et al. Analysis of the upper respiratory tract microbiotas as the source of the lung and gastric microbiotas in healthy individuals. MBio 2015; 6(2): e00037.

39. Charlson ES, Bittinger K, Chen J, et al. Assessing bacterial populations in the lung by replicate analysis of samples from the upper and lower respiratory tracts. PLoS One 2012; 7(9): e42786.

40. Charlson ES, Bittinger K, Haas AR, et al. Topographical continuity of bacterial populations in the healthy human respiratory tract. Am J Respir Crit Care Med 2011; 184(8): 957-63.

41. Erb-Downward JR, Thompson DL, Han MK, et al. Analysis of the lung microbiome in the "healthy" smoker and in COPD. PLoS One 2011; 6(2): e16384.

42. Marsh RL, Kaestli $M$, Chang $A B$, et al. The microbiota in bronchoalveolar lavage from young children with chronic lung disease includes taxa present in both the oropharynx and nasopharynx. Microbiome 2016; 4(1): 37.

43. Segal LN, Alekseyenko AV, Clemente JC, et al. Enrichment of lung microbiome with supraglottic taxa is associated with increased pulmonary inflammation.

Microbiome 2013; 1(1): 19.

44. Segal LN, Clemente JC, Tsay JC, et al. Enrichment of the lung microbiome with oral taxa is associated with lung inflammation of a Th17 phenotype. Nat Microbiol 2016; 1: 16031.

45. Gauguet S, D'Ortona S, Ahnger-Pier K, et al. Intestinal Microbiota of Mice Influences Resistance to Staphylococcus aureus Pneumonia. Infect Immun 2015; 83(10): 4003-14. doi: 10.1128/IAI.00037-15. Epub 2015 Jul 27. 
46. de Steenhuijsen Piters WA, Huijskens EG, Wyllie AL, et al. Dysbiosis of upper respiratory tract microbiota in elderly pneumonia patients. ISME J 2016; 10(1): 97-108. doi: 10.1038/ismej.2015.99. Epub Jul 7.

47. Dickson RP, Erb-Downward JR, Freeman CM, et al. Changes in the lung microbiome following lung transplantation include the emergence of two distinct Pseudomonas species with distinct clinical associations. PLoS One 2014; 9(5): e97214. doi: 10.1371/journal.pone.0097214. eCollection 2014.

48. Flight WG, Smith A, Paisey C, et al. Rapid Detection of Emerging Pathogens and Loss of Microbial Diversity Associated with Severe Lung Disease in Cystic Fibrosis. $J$ Clin Microbiol 2015; 53(7): 2022-9. doi: 10.1128/JCM.00432-15. Epub 2015 Apr 15.

49. Garzoni C, Brugger SD, Qi W, et al. Microbial communities in the respiratory tract of patients with interstitial lung disease. Thorax 2013; 68(12): 1150-6. doi: 10.36/thoraxjnl-2012-202917. Epub 2013 Aug 14.

50. Kawanami T, Yatera K, Yamasaki K, et al. Clinical impact of methicillin-resistant staphylococcus aureus on bacterial pneumonia: cultivation and $16 \mathrm{~S}$ ribosomal RNA gene analysis of bronchoalveolar lavage fluid. BMC Infect Dis 2016; 16:155.(doi): 10.1186/s12879-016-1493-3.

51. Kelly BJ, Imai I, Bittinger K, et al. Composition and dynamics of the respiratory tract microbiome in intubated patients. Microbiome 2016; 4:7.(doi): 10.1186/s40168016-0151-8.

52. Lu H, Qian G, Ren Z, et al. Alterations of Bacteroides sp., Neisseria sp., Actinomyces sp., and Streptococcus sp. populations in the oropharyngeal microbiome are associated with liver cirrhosis and pneumonia. BMC Infect Dis 2015; 15:239.(doi): 10.1186/s12879-015-0977-x.

53. Molyneaux PL, Cox MJ, Willis-Owen SA, et al. The role of bacteria in the pathogenesis and progression of idiopathic pulmonary fibrosis. Am J Respir Crit Care Med 2014; 190(8): 906-13. doi: 10.1164/rccm.201403-05410C.

54. Rogers GB, van der Gast CJ, Cuthbertson L, et al. Clinical measures of disease in adult non-CF bronchiectasis correlate with airway microbiota composition. Thorax 2013; 68(8): 731-7. doi: 10.1136/thoraxjnl-2012-203105. Epub 2013 Apr 6.

55. Shankar J, Nguyen $\mathrm{MH}$, Crespo MM, et al. Looking Beyond Respiratory Cultures: Microbiome-Cytokine Signatures of Bacterial Pneumonia and Tracheobronchitis in Lung Transplant Recipients. Am J Transplant 2016; 16(6): 1766-78. doi: 10.111/ajt.13676. Epub 2016 Feb 10.

56. Toma I, Siegel MO, Keiser J, et al. Single-molecule long-read $16 \mathrm{~S}$ sequencing to characterize the lung microbiome from mechanically ventilated patients with suspected pneumonia. J Clin Microbiol 2014; 52(11): 3913-21. doi:

10.1128/JCM.01678-14. Epub 2014 Aug 20.

57. Willner DL, Hugenholtz P, Yerkovich ST, et al. Reestablishment of recipientassociated microbiota in the lung allograft is linked to reduced risk of bronchiolitis obliterans syndrome. Am J Respir Crit Care Med 2013; 187(6): 640-7. doi: 10.1164/rccm.201209-16800C. Epub 2013 Jan 17.

** This article illustrates how microbiota research could contribute to developing personalized medicine, insofar specific pneumotypes have different effects on health outcomes in patients with different preexisting conditions. 
58. Zakharkina T, Martin-Loeches I, Matamoros S, et al. The dynamics of the pulmonary microbiome during mechanical ventilation in the intensive care unit and the association with occurrence of pneumonia. Thorax 2017.

59. Castro-Nallar E, Shen Y, Freishtat RJ, et al. Integrating microbial and host transcriptomics to characterize asthma-associated microbial communities. BMC Med Genomics 2015; 8: 50.

60. Hasegawa K, Linnemann RW, Mansbach JM, et al. Nasal Airway Microbiota Profile and Severe Bronchiolitis in Infants: A Case-Control Study. Pediatr Infect Dis J 2016: 0000000000001500.

61. Hasegawa K, Mansbach JM, Ajami NJ, et al. Association of nasopharyngeal microbiota profiles with bronchiolitis severity in infants hospitalised for bronchiolitis. Eur Respir J 2016; 48(5): 1329-39. doi: 10.183/13993003.00152-2016. Epub 2016 Oct 6. * This large case-series describes four distinct nasopharyngeal microbiota profiles associated with different risks of severe bronchiolitis in infants.

62. Hyde ER, Petrosino JF, Piedra PA, Camargo CA, Jr., Espinola JA, Mansbach JM. Nasopharyngeal Proteobacteria are associated with viral etiology and acute wheezing in children with severe bronchiolitis. J Allergy Clin Immunol 2014; 133(4): 1220-2. doi: 10.016/j.jaci.2013.10.049. Epub Dec 22.

63. Lu W, Yu J, Ai Q, Liu D, Song C, Li L. Increased constituent ratios of Klebsiella sp., Acinetobacter sp., and Streptococcus sp. and a decrease in microflora diversity may be indicators of ventilator-associated pneumonia: a prospective study in the respiratory tracts of neonates. PLoS One 2014; 9(2): e87504. doi: 10.1371/journal.pone.0087504. eCollection 2014.

64. Mansbach JM, Hasegawa K, Henke DM, et al. Respiratory syncytial virus and rhinovirus severe bronchiolitis are associated with distinct nasopharyngeal microbiota. J Allergy Clin Immunol 2016; 137(6): 1909-13.e4. doi: 10.016/j.jaci.2016.01.036. Epub Apr 6.

65. Perez-Losada M, Castro-Nallar E, Bendall ML, Freishtat RJ, Crandall KA. Dual Transcriptomic Profiling of Host and Microbiota during Health and Disease in Pediatric Asthma. PLoS One 2015; 10(6): e0131819.

66. Pettigrew MM, Gent JF, Kong Y, et al. Association of sputum microbiota profiles with severity of community-acquired pneumonia in children. BMC Infect Dis 2016;

16:317.(doi): 10.1186/s12879-016-1670-4.

67. Sakwinska O, Bastic Schmid V, Berger B, et al. Nasopharyngeal microbiota in healthy children and pneumonia patients. J Clin Microbiol 2014; 52(5): 1590-4. doi: 10.128/JCM.03280-13. Epub 2014 Mar 5.

* This case-control study comparing the nasophayngeal microbiota of children with pneumonia with healthy controls is a nice example of some of the challenges of NGS, in particular, the taxa level of identification. Here, Moraxella lacunata displays opposite association with pneumonia compared to Moraxella catharralis.

68. Wang H, Dai W, Qiu C, et al. Mycoplasma pneumoniae and Streptococcus pneumoniae caused different microbial structure and correlation network in lung microbiota. J Thorac Dis 2016; 8(6): 1316-22. doi: 10.21037/jtd.2016.04.63.

69. Zhou Y, Bacharier LB, Isaacson-Schmid M, et al. Azithromycin therapy during respiratory syncytial virus bronchiolitis: Upper airway microbiome alterations and subsequent recurrent wheeze. J Allergy Clin Immunol 2016; 138(4): 1215-9.e5. doi: 10.016/j.jaci.2016.03.054. Epub May 18. 
70. Nocker A, Richter-Heitmann T, Montijn R, Schuren F, Kort R. Discrimination between live and dead cellsin bacterial communities from environmental water samples analyzed by 454 pyrosequencing. Int Microbiol 2010; 13(2): 59-65.

71. Cangelosi GA, Meschke JS. Dead or alive: molecular assessment of microbial viability. Appl Environ Microbiol 2014; 80(19): 5884-91.

72. Flanagan JL, Brodie EL, Weng L, et al. Loss of bacterial diversity during antibiotic treatment of intubated patients colonized with Pseudomonas aeruginosa. J Clin Microbiol 2007; 45(6): 1954-62.

73. Hofstra JJ, Matamoros $\mathrm{S}$, van de Pol MA, et al. Changes in microbiota during experimental human Rhinovirus infection. BMC Infect Dis 2015; 15:336.(doi): 10.1186/s12879-015-1081-y.

74. Kloepfer KM, Lee WM, Pappas TE, et al. Detection of pathogenic bacteria during rhinovirus infection is associated with increased respiratory symptoms and asthma exacerbations. J Allergy Clin Immunol 2014; 133(5): 1301-7, 7 e1-3.

75. Christie JD, Edwards LB, Kucheryavaya AY, et al. The Registry of the International Society for Heart and Lung Transplantation: twenty-seventh official adult lung and heart-lung transplant report--2010. J Heart Lung Transplant 2010; 29(10): 1104-18.

76. Hayes D, Jr. A review of bronchiolitis obliterans syndrome and therapeutic strategies. J Cardiothorac Surg 2011; 6: 92.

77. Cigana C, Curcuru L, Leone MR, et al. Pseudomonas aeruginosa exploits lipid A and muropeptides modification as a strategy to lower innate immunity during cystic fibrosis lung infection. PLoS One 2009; 4(12): e8439.

78. Mammen MJ, Sethi S. COPD and the microbiome. Respirology 2016; 21(4): 5909.

79. May AK, Brady JS, Romano-Keeler J, et al. A pilot study of the noninvasive assessment of the lung microbiota as a potential tool for the early diagnosis of ventilator-associated pneumonia. Chest 2015; 147(6): 1494-502. doi: 10.378/chest.14687.

80. Kumar PS, Brooker MR, Dowd SE, Camerlengo T. Target region selection is a critical determinant of community fingerprints generated by $16 \mathrm{~S}$ pyrosequencing. PLoS One 2011; 6(6): e20956.

81. Schubert AM, Sinani H, Schloss PD. Antibiotic-Induced Alterations of the Murine Gut Microbiota and Subsequent Effects on Colonization Resistance against Clostridium difficile. MBio 2015; 6(4): e00974.

82. Santee CA, Nagalingam NA, Faruqi AA, et al. Nasopharyngeal microbiota composition of children is related to the frequency of upper respiratory infection and acute sinusitis. Microbiome 2016; 4(1): 34. doi: 10.1186/s40168-016-0179-9. 
We searched PubMed (accessed on February 18 ${ }^{\text {th }}$ 2017) and the reference lists of selected papers to identify studies assessing the association between respiratory microbiota and lower respiratory infections, limited to humans, and with no language restriction. We limited the search to studies using culture-independent techniques to identify and classify bacterial populations in order to increase comparability. We used a combination of search terms as both free and $\mathrm{MeSH}$ terms when applicable, to optimize sensitivity: ((microbiome) OR (microbiota)) AND ((airway*) OR (respiratory tract) OR (nose) OR (nasal) OR (mouth) OR (oral) OR (pharyn*) OR (nasopharyn*) OR (oropharyn*) OR (lung) OR (pulmonar*)) AND ((pneumonia) OR (bronchiolitis) OR (LRTI) OR (lower respiratory tract infection) OR (ARTI) OR (acute respiratory tract infection) OR (ARI) OR (acute respiratory infection)). A total of 125 articles were obtained after excluding animal studies. We screened titles and abstracts, and we retained original papers within the scope of the review.

Table 1. Terminology frequently used in microbiome research

\begin{tabular}{|l|l|}
\hline Terminology & \\
\hline Microbiota & Collection of microorganisms present in a particular site \\
\hline Microbiome & $\begin{array}{l}\text { Collection of microorganisms in a particular site, their genes, } \\
\text { and the surrounding environmental conditions }\end{array}$ \\
\hline Mycobiome & The entire genomic content of fungi in a particular site \\
\hline Virome & $\begin{array}{l}\text { The entire genomic content of viruses in a particular site } \\
\text { establish taxonomic associations, usually by 16S rRNA } \\
\text { sequencing }\end{array}$ \\
\hline Metagenomics & $\begin{array}{l}\text { High-throughput process to characterize the pool of genes from } \\
\text { members of a microbiota, usually by shotgun sequencing }\end{array}$ \\
\hline Metatranscriptomics & $\begin{array}{l}\text { High-throughput process to identify and quantify microbial } \\
\text { genes that are expressed by the microbiota }\end{array}$ \\
\hline Metabolomics & $\begin{array}{l}\text { Study of products of cellular metabolism in a defined } \\
\text { environment, including host's and microbe's metabolites }\end{array}$ \\
\hline $\begin{array}{l}\text { Next-generation } \\
\text { sequencing }\end{array}$ & $\begin{array}{l}\text { In contrast with Sanger sequencing, NGS is based on parallel } \\
\text { sequencing of vast numbers of short reads }\end{array}$ \\
\hline $\begin{array}{l}\text { Whole-genome } \\
\text { Shotgun sequencing }\end{array}$ & $\begin{array}{l}\text { Method to characterize whole genomes by sequencing a large } \\
\text { number of random small DNA fragments in parallel and } \\
\text { assembling them into larger contigs }\end{array}$ \\
\hline $\begin{array}{l}\text { 16S rRNA gene } \\
\text { sequencing }\end{array}$ & $\begin{array}{l}\text { Method to characterize bacterial populations by sequencing } \\
\text { variable regions of the highly conserved bacterial 16S rRNA } \\
\text { gene }\end{array}$ \\
\hline $\begin{array}{l}\text { Operational } \\
\text { Taxonomic Unit } \\
\text { as a surrogate for taxa in metataxonomics studies. 97\% }\end{array}$ \\
\hline
\end{tabular}




\begin{tabular}{|l|l|}
\hline (OTU) & similarity is commonly used as cut-off for species surrogate \\
\hline Abundance & Total number of bacterial individuals in a sample \\
\hline Richness & Number of different species/OTUs present in a sample \\
\hline Evenness & $\begin{array}{l}\text { Measure of similarity in relative abundance / frequency } \\
\text { distribution of OTUs within a community. Dominance of one } \\
\text { taxa implies decreased evenness }\end{array}$ \\
\hline Alpha Diversity & $\begin{array}{l}\text { Measure of diversity within a sample, taking into account } \\
\text { richness and/or evenness. Commonly used metrics of } \alpha \text { - } \\
\text { diversity include Chao1, Shannon and Simpson indexes }\end{array}$ \\
\hline Chao 1 Index & Measure of observed and unobserved richness \\
\hline Shannon Index & $\begin{array}{l}\text { Measure of diversity combining richness and evenness. Higher } \\
\text { values indicate higher diversity }\end{array}$ \\
\hline Simpson Index & $\begin{array}{l}\text { Measure of dominance (complimentary measure of evenness). } \\
\text { Higher values indicate lower evenness }\end{array}$ \\
\hline Beta Diversity & $\begin{array}{l}\text { Measure of diversity between samples. High } \beta \text {-diversity implies } \\
\text { large dissimilarities between samples }\end{array}$ \\
\hline
\end{tabular}


Table 2. Overview of study characteristics included in the systematic review (Adults)

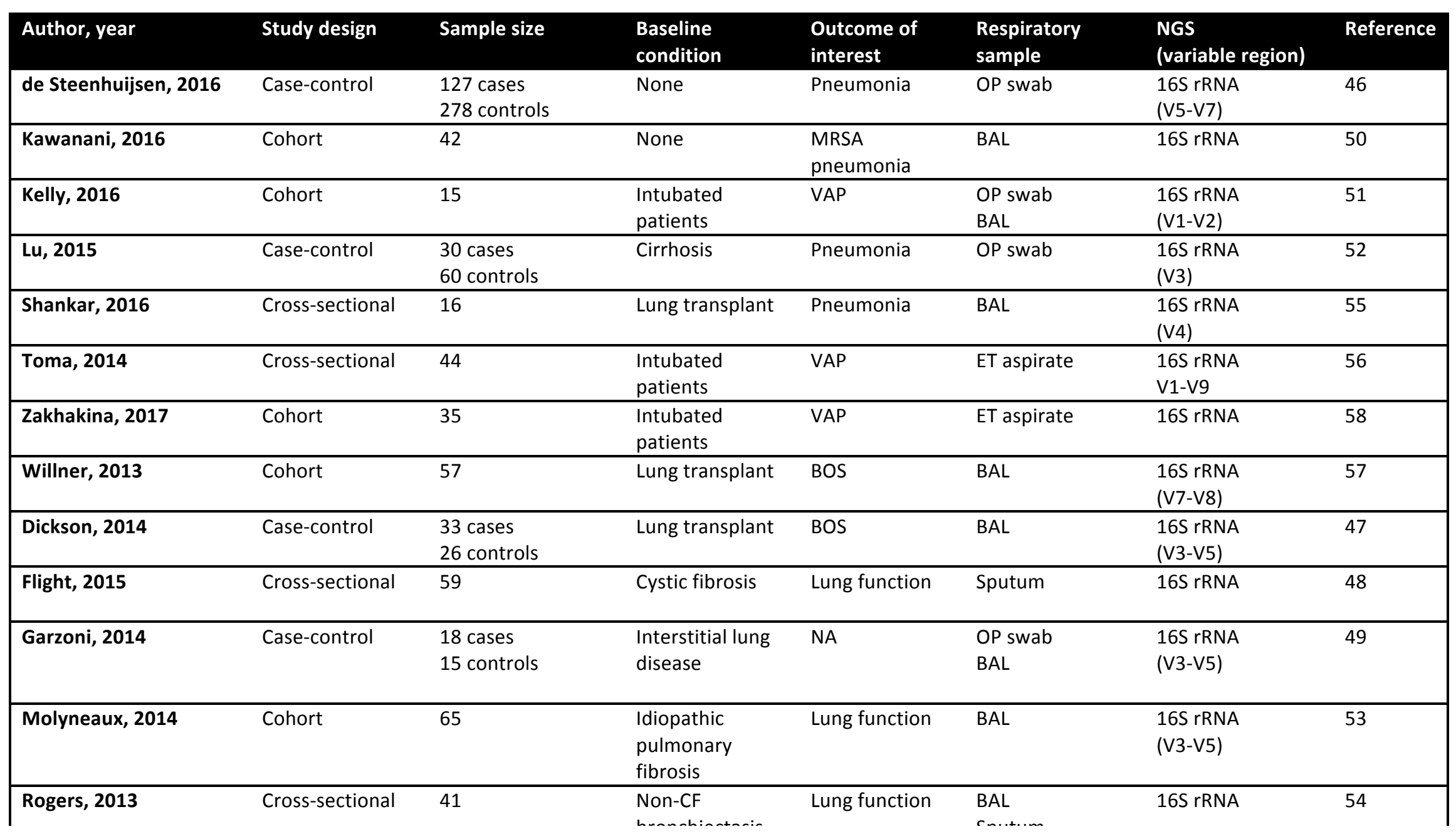


Table 3. Overview of study characteristics included in the systematic review (Children)

\begin{tabular}{|c|c|c|c|c|c|c|c|}
\hline Author, year & Study design & Sample size & Baseline condition & $\begin{array}{l}\text { Outcome of } \\
\text { interest }\end{array}$ & $\begin{array}{l}\text { Respiratory } \\
\text { sample }\end{array}$ & $\begin{array}{l}\text { NGS (variable } \\
\text { region) }\end{array}$ & Reference \\
\hline Lu, 2014 & Cross-sectional & 25 & $\begin{array}{l}\text { Intubated } \\
\text { newborns }\end{array}$ & VAP & ET aspirate & $\begin{array}{l}\text { 16S rRNA } \\
\text { (V3) }\end{array}$ & 63 \\
\hline Pettigrew, 2016 & Cohort & 383 & Pneumonia & Severity & $\begin{array}{l}\text { Sputum } \\
\text { NP swab } \\
\text { OP swab }\end{array}$ & $\begin{array}{l}\text { 16S rRNA } \\
\text { (V4) }\end{array}$ & 66 \\
\hline Sakwinska, 2014 & Case-control & $\begin{array}{l}50 \text { cases } \\
50 \text { controls }\end{array}$ & None & Pneumonia & NP swab & $\begin{array}{l}\text { 16S rRNA } \\
\text { (V1-V2) }\end{array}$ & 67 \\
\hline Wang, 2016 & Case-control & $\begin{array}{l}21 \text { cases } \\
11 \text { controls }\end{array}$ & None & Pneumonia & BAL & $\begin{array}{l}\text { 16S rRNA } \\
\text { (V3-V4) }\end{array}$ & 68 \\
\hline $\begin{array}{l}\text { Castro-Callar, } \\
2015\end{array}$ & Case-control & $\begin{array}{l}8 \text { cases } \\
6 \text { controls }\end{array}$ & None & Asthma & Nasal brush & $\begin{array}{l}\text { Whole genome } \\
\text { shotgun }\end{array}$ & 59 \\
\hline Hasegawa, 2016 & Case-control & $\begin{array}{l}40 \text { cases } \\
110 \text { controls }\end{array}$ & None & Bronchiolitis & Nasal swab & $\begin{array}{l}\text { 16S rRNA } \\
\text { (V4) }\end{array}$ & 60 \\
\hline Hasegawa, 2016 & Cross-sectional & 1005 & Bronchiolitis & ICU admission & NP aspirate & $\begin{array}{l}\text { 16S rRNA } \\
\text { (V4) }\end{array}$ & 61 \\
\hline Hyde, 2014 & Cross-sectional & 100 & Bronchiolitis & NA & NP aspirate & $\begin{array}{l}\text { 16S rRNA } \\
\text { (V3-V5) }\end{array}$ & 62 \\
\hline Mansbach, 2016 & Cross-sectional & 740 & Bronchiolitis & NA & NP aspirate & $\begin{array}{l}\text { 16S rRNA } \\
\text { (V4) }\end{array}$ & 64 \\
\hline Teo, 2015 & Cohort & 234 & High risk of atopy & $\begin{array}{l}\text { Asthma } \\
\text { LRI }\end{array}$ & NP aspirate & $\begin{array}{l}\text { 16S rRNA } \\
\text { (V4) }\end{array}$ & 19 \\
\hline Zhou, 2016 & Cohort & 39 & Bronchiolitis & $\begin{array}{l}\text { Recurrent } \\
\text { wheezing }\end{array}$ & Nasal lavage & $\begin{array}{l}\text { 16S rRNA } \\
\text { (V1-V3) }\end{array}$ & 69 \\
\hline
\end{tabular}

VAP: ventilator-associated pneumonia; ET: endotracheal; NP: nasopharyngeal; OP: oropharyngeal; BAL: bronchoalveolar lavage; ICU: intensive care unit; LRI: lower respiratory infection 